\title{
COVID-19 vaccination in patients with immunity-mediated kidney disease
}

\author{
Maria Prendecki ${ }^{1,2 凶}$, Michelle Willicombe ${ }^{1,2 凶}$ and Stephen P. McAdoo $\mathbb{1}^{1,2}$ \\ Vaccination against SARS-CoV-2 seems to be safe in patients with immunity-mediated kidney \\ disease, although their immunological responses to vaccination are impaired. Further strategies, \\ including the administration of additional vaccine doses and passive immunization with \\ long-acting monoclonal antibodies, might increase protection in this vulnerable patient group.
}

Patients
treated with
B cell-depleting
agents such
as rituximab
show markedly
diminished
serological
responses

${ }^{1}$ Centre for Inflammatory Disease, Department of Immunology and Inflammation, Imperial College London, London, UK.

${ }^{2}$ Imperial College Renal and Transplant Centre, Imperial College Healthcare NHS Trust, Hammersmith Hospital, London, UK.

凶e-mail:m.prendecki@ imperial.ac.uk; m.willicombe08@ imperial.ac.uk
Chronic kidney disease increases the risk of severe disease and mortality following infection with SARS-CoV-2 and, in patients with immunity-mediated kidney disease, treatment with immunosuppressants is an additional risk factor for poor outcomes ${ }^{1}$. However, these patients were excluded from all major COVID-19 vaccine trials and, thus, our understanding of vaccine safety, immunogenicity and efficacy in these patients is limited.

The safety profile of current COVID-19 vaccines was established in rapidly co-ordinated phase II and III clinical trials, and was further confirmed during their global rollout. Myocarditis and thrombotic thrombocytopenia emerged as very rare complications associated with mRNA and adenoviral vector vaccines, respectively ${ }^{2}$. No data suggest that a prior history of autoimmune disease increases the risk of these complications.

Although epidemiological studies do not indicate a significant causal relationship, vaccine-triggered de novo or relapsing autoimmunity is a common concern for patients and several case reports have documented de novo glomerulonephritis after SARS-CoV-2 vaccination, including ANCA-associated vasculitis, minimal change disease and IgA nephropathy ${ }^{3}$. However, despite the involvement of inflammatory pathways associated with autoimmunity in the protective immune response induced by COVID-19 vaccines, reports of vaccination outcomes in large cohorts have not identified a post-vaccination increase in the relapse rate of patients with chronic inflammatory diseases ${ }^{4}$. Moreover, the benefit of vaccination for preventing severe COVID-19 versus the theoretical risk of a treatable disease flare favours vaccination.

The responses to vaccination in patients with immunity-mediated kidney disease are known to be affected by the use of immunosuppressive treatments; for example, humoral responses to influenza and pneumococcal vaccination are impaired by rituximab therapy ${ }^{5}$. The underlying disease and patient characteristics, such as age and co-morbidities, as well as vaccine type, also affect the quality of the immune response. A meta-analysis of the immune responses of healthy
SARS-CoV-2 vaccine trial participants found that mRNA vaccination elicits greater serological responses than adenoviral vector vaccines, which seem to induce more robust $\mathrm{T}$ cell responses ${ }^{6}$. The immune correlates of protection from COVID-19 have yet to be defined in both healthy and immunosuppressed populations, but full disease protection probably requires a combination of humoral and cellular responses. A study in non-human primates, for example, suggested that $\mathrm{CD} 8^{+}$ $\mathrm{T}$ cell responses have an important protective role in the context of waning humoral immunity ${ }^{7}$.

Although studies of patients with immunity-mediated kidney disease are limited ${ }^{8}$, several reports have assessed the response to SARS-CoV-2 vaccination in patients receiving immunosuppression for rheumatic and other chronic inflammatory conditions. In most cohorts, the majority of patients demonstrate seroconversion the largest study to date reported $86 \%$ seroconversion following two doses of the BNT162b2 mRNA vaccine in 686 individuals receiving treatment for autoimmune rheumatic disease ${ }^{4}$. However, studies consistently show that the magnitude of the immunological response is blunted compared with the general population and that treatment-related effects depend on the immunosuppressive agent used.

Patients treated with B cell-depleting agents such as rituximab show markedly diminished serological responses to both mRNA-based and adenoviral vector-based SARS-CoV-2 vaccines. The serological response correlates positively with B cell count and time elapsed since rituximab treatment ${ }^{4,8,9}$. In one study, only $41 \%$ of patients treated with rituximab in the preceding 6 months had a detectable serological response ${ }^{8}$. Of note, $\mathrm{T}$ cell responses were preserved in this group, although whether they are sufficient to protect serological non-responders from severe disease remains unknown.

Similar to other vaccines, treatment with high-dose glucocorticoids ( $>10 \mathrm{mg} /$ day) is associated with low serological responses to SARS-CoV-2 mRNA vaccines ${ }^{9}$. In a study that included patients with systemic lupus erythematosus and vasculitis, treatment with mycophenolate 
additional vaccine doses might ... enhance immunogenicity in most patients with autoimmune kidney disease mofetil was also associated with an impaired humoral response to mRNA vaccination ${ }^{4}$. Conversely, calcineurin inhibitors had a modest effect on $\mathrm{T}$ cell responses and no effect on serological responses in a cohort of patients with glomerular disease ${ }^{8}$.

Additional studies have assessed the effects of anticytokine therapies (for example, anti-TNF), and of conventional and targeted synthetic disease-modifying antirheumatic drugs (for example, methotrexate) in cohorts of patients with rheumatic diseases. However, these agents are not commonly used to treat glomerulonephritis, which highlights the need for studies focused on patients with glomerular disease, who are typically treated with different agents, and in whom the underlying kidney dysfunction might compound iatrogenic immunosuppression.

Several strategies are currently being investigated to optimize vaccine efficacy and provide additional protection to patients treated with immunosuppressants. Early studies in kidney transplant recipients reported that patients who had previously not responded to routine two-dose vaccination schedules had immunological responses to a third vaccine dose. Additional doses of the same vaccine and heterologous prime-boost regimens have been investigated but comparative data are not yet available. We expect that additional vaccine doses might also enhance immunogenicity in most patients with autoimmune kidney disease, although they might be insufficient to overcome the profound effect of B cell depletion on humoral responses. The additional booster approach is already recommended by some public health authorities and data from non-transplant populations should be forthcoming.

The timing of vaccination, including of booster doses, must also be considered. For example, delaying vaccination until around 4 weeks prior to the next rituximab infusion cycle has been suggested for patients receiving regular rituximab infusions. Although emerging data indicate that this approach might maximize the immune response, delaying primary vaccination when community transmission rates are high might not be ethical. However, scheduling booster doses following completion of rituximab cycles or when B cell reconstitution has occurred might be feasible. In some cases, deferring non-urgent rituximab retreatment for short periods to provide an opportunity for vaccination or booster administration (for example, in patients with stable disease receiving routine maintenance infusions and at low risk of relapse) might be also be safe. Of note, in patients with immunity-mediated kidney disease treated with oral immunosuppressants, temporarily withholding medications around the time of vaccination routinely might be inappropriate owing to the risks and consequences of a disease flare (for example, nephrotic relapse or progression to kidney failure), which are likely to be greater than those in patients with other rheumatic and inflammatory diseases.

Passive immunization with monoclonal antibodies (mAbs) against SARS-CoV-2 might be a prophylactic option in patients lacking serological responses to vaccination. Top-line data from the PROVENT study (NCT04625725), which investigated a long-acting mAb as pre-exposure prophylaxis in clinically vulnerable patients, show $77 \%$ efficacy in preventing symptomatic COVID-19 at 183 days following a single vaccine dose, compared with placebo. However, some have suggested that the administration of SARS-CoV-2 mAbs might impair future vaccine responses by interacting with vaccine-generated cognate antigens.

New vaccines continue to be developed and some might improve vaccination outcomes in patients treated with immunosuppression. Mucosal vaccines against SARS-CoV-2, for example, induced a robust dimeric secretory IgA response, long-lasting tissue-resident cellular responses and enhanced viral clearance in the upper respiratory tract of non-human primates compared with controls immunized intramuscularly ${ }^{10}$. The effect of systemic immunosuppression on the response to these vaccine types might be less substantial than that observed with current vaccine options.

Overall, SARS-CoV-2 vaccines are safe and immunogenic in most patients with immunity-mediated kidney disease, although the overall immune response is impaired and rituximab, in particular, has a profound effect on serological responses to vaccination. This immune impairment is likely to reduce vaccine-induced protection from disease, and studies now need to define the clinical efficacy of these vaccines and the laboratory correlates of protection for this group of patients. Most immunogenicity studies have focused on serological responses to mRNA vaccines and more work is needed to understand the effect of immunosuppression on adenoviral vector vaccines and, for all vaccine types, to clarify the induction and clinical importance of $\mathrm{T}$ cell responses in patients treated with immunosuppressants. Additional vaccine strategies to protect patients with immunosuppression are under investigation and should complement the ongoing recommendations that these patients maintain social distancing and the prioritization of vaccination for their household members.

1. Gianfrancesco, M. et al. Characteristics associated with hospitalisation for COVID-19 in people with rheumatic disease: data from the COVID-19 Global Rheumatology Alliance physicianreported registry. Ann. Rheum. Dis. 79, 859-866 (2020).

2. UK MHRA. Coronavirus vaccine - weekly summary of Yellow Card reporting. https://www.gov.uk/government/publications/coronavirus covid-19-vaccine-adverse-reactions/coronavirus-vaccine-summaryof-yellow-card-reporting (2021).

3. Tan, H. Z. et al. Is COVID-19 vaccination unmasking glomerulonephritis? Kidney Int. 100, 469-471 (2021).

4. Furer, V. et al. Immunogenicity and safety of the BNT162b2 mRNA COVID-19 vaccine in adult patients with autoimmune inflammatory rheumatic diseases and in the general population: a multicentre study. Ann. Rheum. Dis. 80, 1330-1338 (2021).

5. Oren, $\mathrm{S}$. et al. Vaccination against influenza in patients with rheumatoid arthritis: the effect of rituximab on the humoral response. Ann. Rheum. Dis. 67, 937-941 (2008).

6. McDonald, I., Murray, S. M., Reynolds, C. J., Altmann, D. M. \& Boyton, R. J. Comparative systematic review and meta-analysis of reactogenicity, immunogenicity and efficacy of vaccines against SARS-CoV-2. NPJ Vaccines 6, 74 (2021).

7. McMahan, K. et al. Correlates of protection against SARS-CoV-2 in rhesus macaques. Nature 590, 630-634 (2021).

8. Prendecki, M. et al. Humoral and T-cell responses to SARS-CoV-2 vaccination in patients receiving immunosuppression. Ann. Rheum. Dis. 80, 1322-1329 (2021).

9. Deepak, P. et al. Effect of immunosuppression on the immunogenicity of mRNA vaccines to SARS-CoV-2: A prospective cohort study. Ann. Intern. Med. https://doi.org/10.7326/M21-1757 (2021).

10. Sui, Y. et al. Protection against SARS-CoV-2 infection by a mucosal vaccine in rhesus macaques. JCI Insight 6, e 148494 (2021).

\section{Competing interests}

M.W. has received support to use the T-SPOT ${ }^{\circledR}$ Discovery SARS-CoV-2 by Oxford Immunotec. The other authors declare no competing interests. 